\title{
Declines In Chill Coma Recovery and Negative Geotaxis Due To an Entamopathogen in Drosophila melanogaster the Fruit Flies
}

\author{
Usha Bhocal ${ }^{1}$, Bijender Singh Sabharwal ${ }^{2}$ and Veer Bhan ${ }^{3}$ \\ ${ }^{I}$ Research Scholar, Depatment of Biotechnology, University Institute of Engineering and Technology, \\ Maharshi Dayanand University, Rohtak, Haryana, India \\ ${ }^{2}$ P.G. Student, Depatment of Law, Maharshi Dayanand University, Rohtak, Haryana, India \\ ${ }^{3}$ Assistant Professor, Depatment of Biotechnology,University Institute of Engineering and Technology, \\ Maharshi Dayanand University,Rohtak-124001 Haryana, India
}

\begin{abstract}
Infection related studies in Drosophila melanogaster provide insight into both mechanisms of host resistance and pathogens tolerance. The advantages of the fly as an experimental system include its genetic tractability, short life span and the observable possibility and to quantitatively analyse behavioral responses. At present, research has been limited by the relatively few metrics that can be used to measure health and illness during the course of infection. Here by, we explain the pleiotropic measurements of infection-related declines in flies performance on two different assays. D. melanogaster are somewhat slower to recover from an induced chill coma after fungal infection with Beauveria bassiana. Due to fungal infection, flies performance also gets affected during a negative geotaxis assay, revealing a decrease in their behavioral response. In addition, to provide new advancements for assessing health, these non-lethal assays also suggest many pathological consequences and also up and downs of metabolic that may occur over the entire course of an infection.
\end{abstract}

Keywords: Chill coma, Drosophila, geotaxis, infection, resistance

\section{Introduction}

All organisms need to defend themselves against pathogens, parasites and other natural enemies, and investment in defense is a significant component of the life history strategy of most animals and plants [1,2]. Infection can impact the health of an organism in complex ways. Direct damage to the host caused by pathogenic toxins, damage to host tissue resulting from immune effectors, and the energetic expense of responding to an infection are all known to affect the health of a host $[3,4,5,6]$. Specifically, D.melanogaster possess thermo sensitivity (the ability to detect temperature change), allowing individuals to seek out desirable habitat $[7,8]$ note that the strength of thermo sensitivity a species elicits is dependent on the species surrounding environment. Surviving an infection requires both resistance, the ability to limit pathogen burden, and tolerance, the ability to minimize the impact a pathogen has on fitness $[3,9,10]$.Also,insects have evolved a range of molecular adaptations to cope with seasonal exposure to stressful (high or low) temperatures [11].

Understanding the various pathways mediating resistance and tolerance allows for better development of interventions that focus on the maintenance of health throughout an infection. However, dissecting the mechanisms that determine the balance of eliminating pathogens and the damage and energetic cost of mounting that immunological response requires metrics that move beyond survival and allow assessment of health during infection.

Work with the model organism Drosophila melanogaster has provided important insights into the roles of different molecular pathways involved in immunity[12,13,14]. D. melanogaster provides a genetically tractable system where a large numbers of replicates can be assessed while still being a whole organism system with biological complexity. Additionally, a relationship between infection, immunity, and reproductive fitness in insects has been well established. In environments where nutrients are limited, there is a negative correlation between female fecundity and resistance to bacterial infection in D. melanogaster [15]. Additionally, immune challenged females not only have fewer offspring, but those offspring also have shorter lifespans compared to the offspring of unchallenged female D. melanogaster [16]. The molecular mechanisms behind recovery from cold stress are complex and it seems that more genes/proteins are activated during the recovery phases than during the period of the cold stress itself $[17,18]$.

Here we report on two distinct assays that are not measures of natural declines in infection, but rather measure the flie's ability to recover from stress or react to stimuli. Such assays are commonly used in the field of aging research in D.melanogaster, which has long been recognized that measures of both health span and longevity are critical to understanding the biology of aging. We hypothesized that these metrics could also be used to assess health during infection because D.melanogaster shows an age-related up-regulation of inflammatory genes and expression patterns that characterize aging and the induction of an immune response in 
these animals are related [19]. Additionally,in aged flies as well,the wasting and loss of circadian rhythms reported to occur during infection [20,21].

Here we show infection-related deficits for the two behavioral assays, chill coma recovery and negative geotaxis. When low temperature exposures were given to insects, they enter into a reversible period of immobility referred to as chill coma. While the physiological causes behind induction and recovery from this coma are incompletely understood, the duration of time taken by $D$. melanogaster to be able to stand after returning to the warmer temperature is altered by the animal's environment prior to cold exposure, its energy accumulation sites, and its age related consequences[22,23,24,25]. Negative geotaxis is the measure of how quickly a fly is able to vertically climb after being tapped to the bottom of a vessel as part of its innate escape response. Negative geotaxis is measured by either the climbed distance in a set time or the length of time it takes an animal to climb a set distance. Negative geotactic ability has been shown to be sensitive to oxidative stress, age related parameters, and previous exposure of cold, but not to fungal infection $[26,27,28,29]$. We finally hypothesize that the changes in physiological parameters that causes performance deficits in both of these pleiotropic assays are somehow affected by fungal infections, such that both assays can be used during fungal infection to detect decreases in different health consequences.

\section{Materials and Methods}

\subsection{Collection and maintenance of flies}

Drosophila species individuals will be collected along an altitudinal range $(219 \mathrm{~m}$ to $2202 \mathrm{~m})$ in a single planned collection trip to avoid seasonal variations. Before collection, climatic and geographical data of $8-10$ sites will be analyzed and 4-5 sites, which will be showing a clinal trend of $\mathrm{T}_{\mathrm{cv}}$ and $\mathrm{RH}_{\mathrm{cv}}$ will be selected. Temperature of collection site will be noted down at the time of collection and the methodology of experiment will be followed according to the temperature of collection site. Large and random samples will be made i.e. 3 or 4 places from same site. Agar-sugar food vials will be used for collection.Trap bait method and net sweeping method will be used to procure a maximum number of flies of a particular species as well as different species with minimum expenditure of time and material.

After identification of the naturally collected flies, males and females will be separated and isofemale lines will be established with the wild caught females. Ten isofemale lines in three replicates per population will be established. The experiments will be performed at $25^{\circ} \mathrm{C}, 12 \mathrm{~h} \mathrm{~L}$ : D in a humidified incubator.

\subsection{Preparation of fungal material}

B. bassiana strain will be stored as a stock spore suspension (105 spores per $\mathrm{ml})$ in $25 \%$ glycerol at $-80^{\circ} \mathrm{C}$.Cultures will be generated from this stock by spreading $5 \mu$ of the solution onto Petri dishes with Potato dextrose agar (PDA) and culturing for $3-4$ weeks at $29 \pm 0.5^{\circ} \mathrm{C}$. These plates will be then used to culture the next set of Potato dextrose agar plates (inoculated by streaking with a nichrome wire loop), which when mature (after 3-4 weeks) will be used in the experiments. Before use, to make sure the fungus colonies are mature and sporulating, samples from all plates will be examined under a microscope.

\subsection{Infection mode}

Natural infection by entomopathogenic fungi. Anesthetized flies were shaken for a few minutes in a petri dish containing a sporulating fungal culture. Flies covered by spores were then removed to fresh Drosophila medium and incubated at $25^{\circ} \mathrm{C}$. Fungi are important insect pathogens and spores of some entomopathogenic species such as B. bassiana can sporulate on the cuticle and produce chitinases and proteases to penetrate the insect.

In this study, we infected Drosophila melanogaster adults on sex basis aged 3-4 days at $25^{\circ} \mathrm{C}$.Natural infection was initiated by shaking anesthetized flies in a petridish containing a sporulating culture of Beauveria bassiana was incubated at $25^{\circ} \mathrm{C}$ for required time period.

\subsection{Chill Coma Recovery}

Every day a new group of infected and control flies were transferred to clean glass vials ( 50 flies per vial) without anesthesia around noon and placed for three hours in melting ice in a bucket to induce chill coma. After that in a large petri plate flies were kept. A timer was started once the flies were placed in petriplates and only those flies were considered recovered only when they were able to stand on their legs.It should be noted that a single set of flies was not used throughout the course of infection experiment due to the reported effect of exposure to cold on longevity and immunity [30,31].Calculation is done by taking mean and standard deviation respectively. 


\subsection{Negative Geotaxis}

Negative geotaxis is a frequently used index of locomotor behavior in flies $[32,28,33,34,35,36,37,38,39,40]$. Additionally, the negative geotaxis assay was performed in a manner similar to that published by [39]. 50 infected and 50 control flies were transferred once a day in the early afternoon without anesthesia into $10 \mathrm{~cm}$ tall clean glass vials. The glass vials were placed in a rectangular frame to keep them upright and put in front of a light box at room temperature in a chamber kept between $50 \%$ and $60 \%$ humidity. Waited for ten minutes so that all flies after transfer become fully awake and active. Individuals that did not become active were removed after waiting for an additional five minutes. After the vial was tapped three times and climbing time was recorded by a stop watch as $10 \mathrm{sec}, 30 \mathrm{sec}, 60 \mathrm{sec}$ post startle. All work was done on sex basis. Flies were then transferred without anesthesia back into their rearing vial.

\subsection{Infection impairs chill coma recovery}

\section{Results}

Beauveria bassiana infection impairs chill coma recovery. Experiments were done on D. melanogaster that have previously been reported to undergo infection-related changes in circadian rhythms, display sickness induced anorexia, and have revealed different infection-dependent and background-dependent resistance and tolerance mechanism [41,42 unpublished observation].To measure chill coma recovery, flies were placed on ice for three hours to induce a chill coma. Flies were then returned to room temperature and monitored for recovery as determined by their ability to stand on their legs. Both fungal infected flies showed delay in time to recover. Male flies recovered late in control as well as in infected flies.

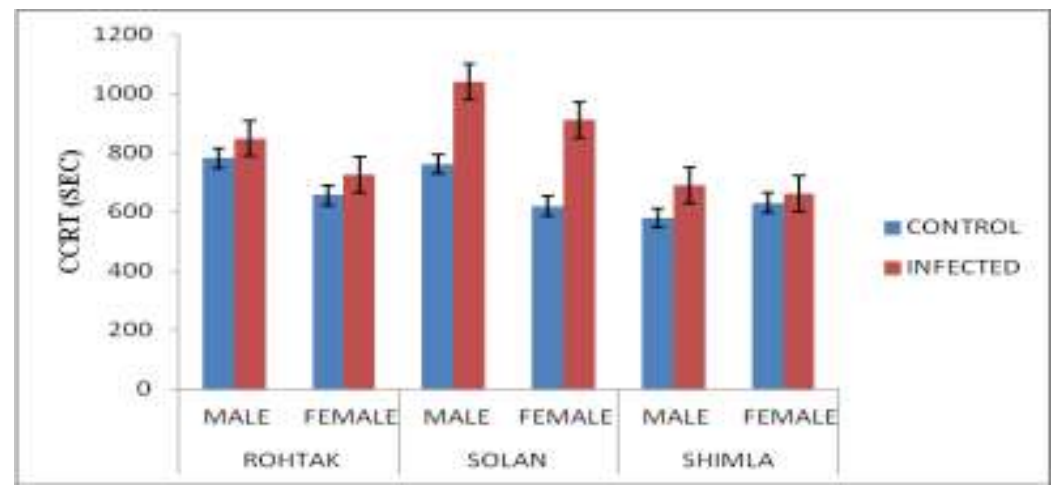

Figure 1 Effect of B. bassiana infection on the mean CCRT (chill coma recovery time) of D. melanogaster collected from three different sites (Rohtak, Solan and Shimla) at $25^{\circ} \mathrm{C}$.Values are expressed as mean $\pm \mathrm{SD}$ $(\mathrm{N}=50)$.

\subsection{Infection declines in negative geotaxis}

Flies showed declines in the height climbed at $10 \mathrm{sec}, 30 \mathrm{sec}$ and $60 \mathrm{sec}$ post-startle during infection as compared to control. Comparison of the data by infected versus control flies showed overall significant differences. After infection flies locomotory ability was decreased and they took more time to cover the required distance.

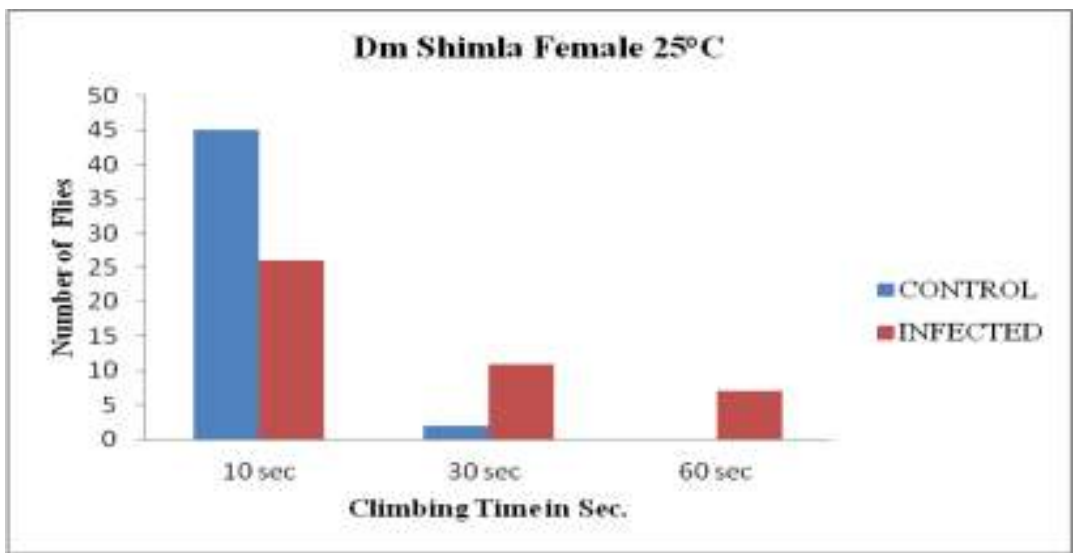

Figure 2(a) B.bassiana infection impairs climbing ability of D.melanogaster of Shimla female flies,time interval in seconds (10sec,30 sec and $60 \mathrm{sec}),(\mathrm{N}=50)$ 


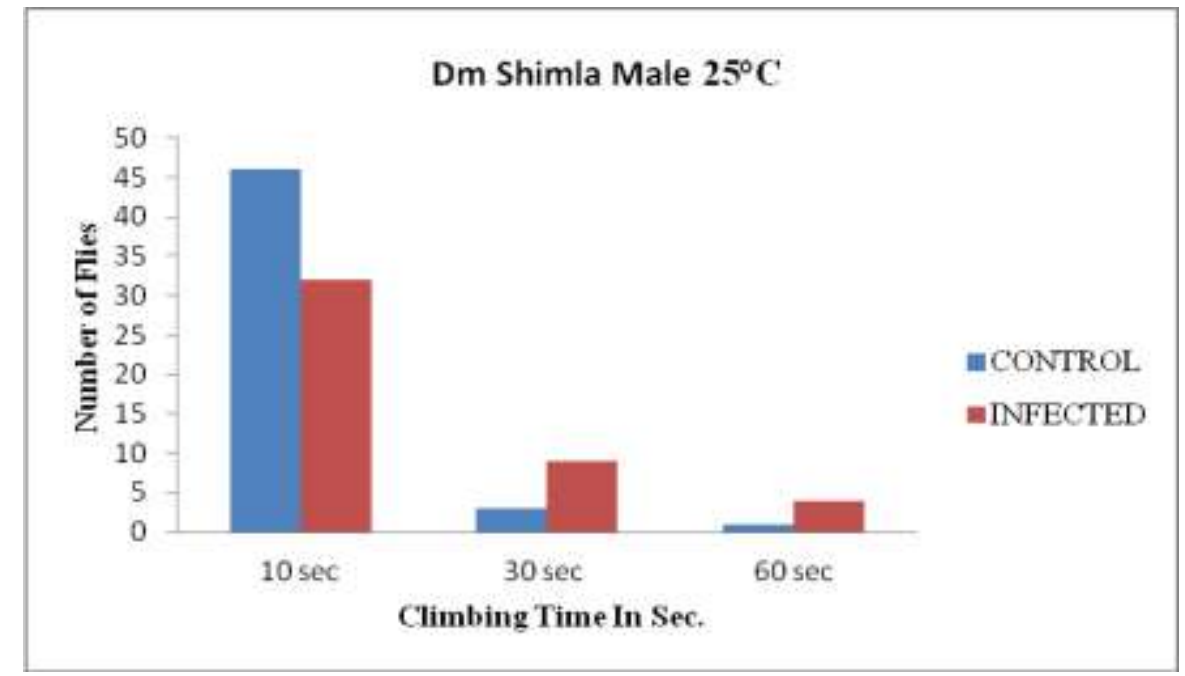

Figure 2(b) B.bassiana infection impairs climbing ability of D.melanogaster of Shimla male flies, time interval in seconds (10sec,30 sec and $60 \mathrm{sec}),(\mathrm{N}=50)$

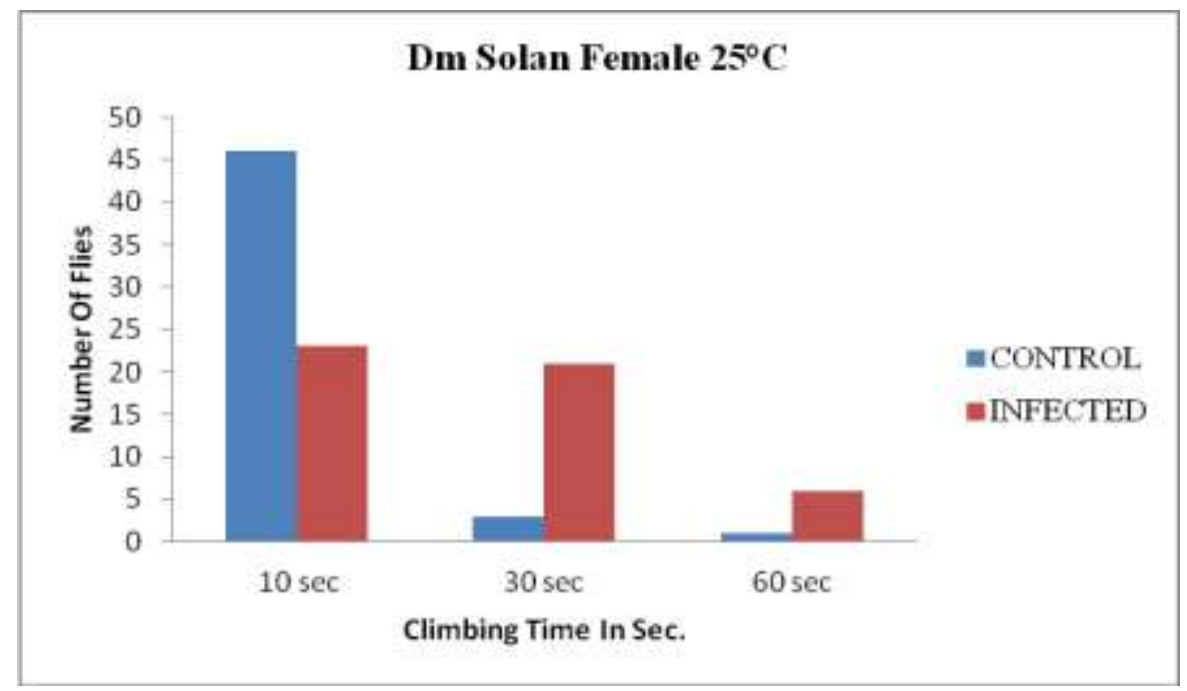

Figure 2(c) B.bassiana infection impairs climbing ability of D.melanogaster of Solan female flies, time interval in seconds (10 sec,30 sec and $60 \mathrm{sec}),(\mathrm{N}=50)$

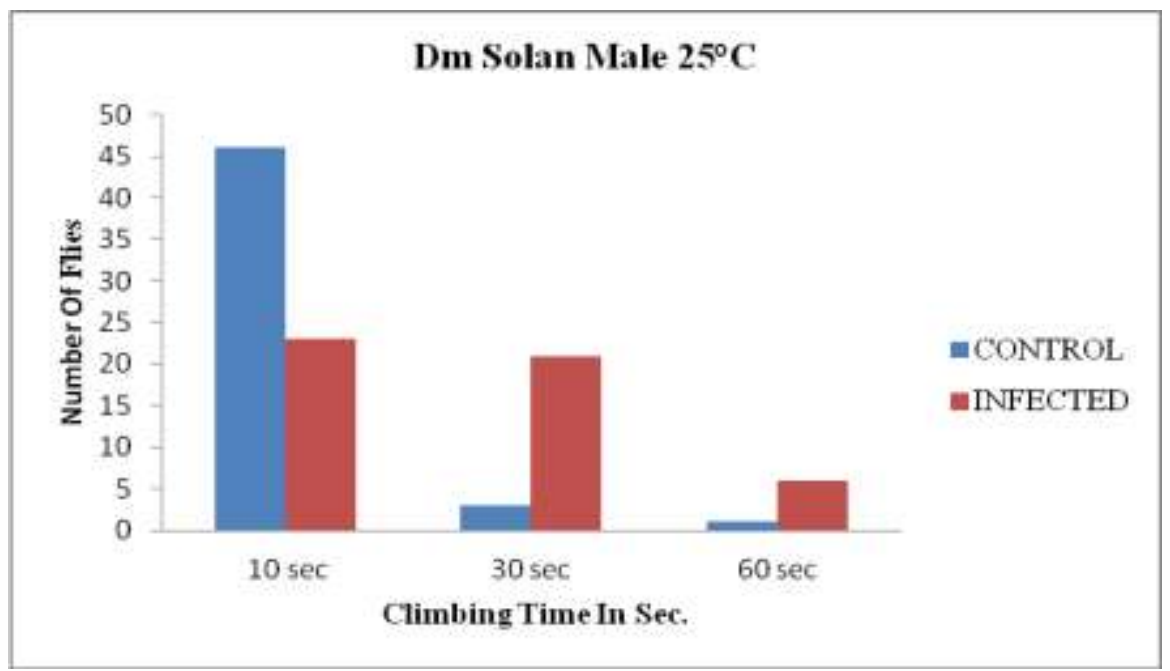

Figure 2(d) B.bassiana infection impairs climbing ability of D.melanogaster of Solan male flies,time interval in seconds (10 sec,30 sec and $60 \mathrm{sec}),(\mathrm{N}=50)$ 


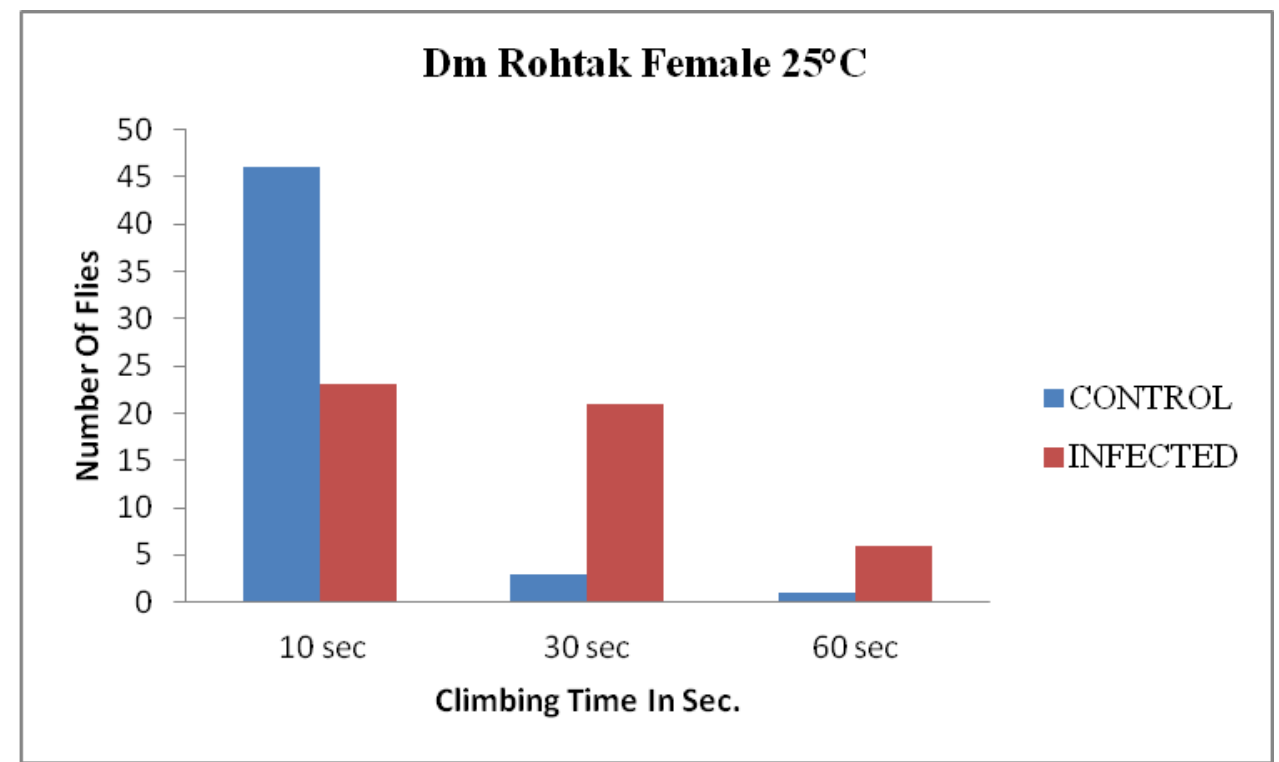

Figure 2(e) B.bassiana infection impairs climbing ability of D.melanogaster of Rohtak female flies,time interval in seconds (10sec,30 sec and $60 \mathrm{sec}),(\mathrm{N}=50)$

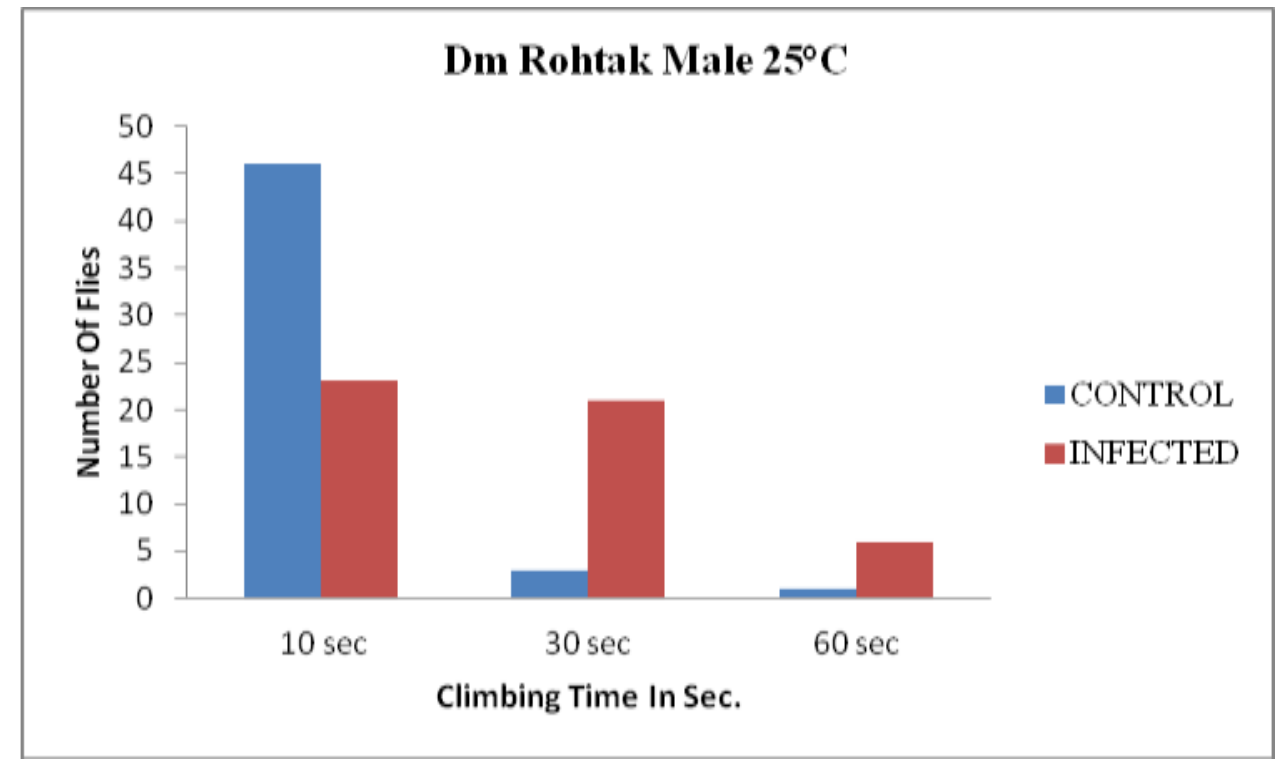

Figure 2(f) B.bassiana infection impairs climbing ability of D.melanogaster of Rohtak male flies,time interval in seconds (10sec,30 sec and $60 \mathrm{sec}),(\mathrm{N}=50)$

\section{Discussion}

Chill coma temperature for cold tolerance and distribution has been used in many studies and has been shown to be a good proxy. Earlier methods generally relied on behavioural criteria, defining chill-coma temperature at which the animals became motionless, unresponsive to stimulation, or fell over because they had lost control over the righting reflex. Drosophila melanogaster, the fruit fly, has been used to study molecular mechanisms of a wide range of human diseases such as cancer, cardiovascular disease and various neurological diseases [43].These behavioral assays are widely applicable for studying the role of genetic and environmental factors on fly behavior. Here we demonstrate a significant impairment in chill coma recovery during Beauveria bassiana infection. Chill coma recovery is a process that is known to have both genetic and environmental influences [44].

In certain insects including $D$. melanogaster, it has been shown that expression of immune response genes are increased in response to long term or repeated exposure to cold suggesting potential shared responses to cold and infection [45,46].Similarly,the genes that are up-regulated during infection were also resulted in upregulation of more general stress response genes after chill coma recovery assay.The heat shock proteins, particularly Hsp70, when upregulated is known to be of functional importance in response to both stresses $[47,48,49]$, suggesting some shared protective mechanisms. The Frost gene likewise plays a significant role in 
chill coma recovery [47] and is known to be up-regulated during infection [50].These may highlight the importance of protection against cellular damage to survival of each of these stresses. The combined impact of chill and infections may overwhelm the protective mechanisms of these flies, resulting in delayed chill coma recovery or even damage resulting in death. It is also likely that relationship between the physiology of infection and delayed chill coma recovery is due to the metabolic changes that occur during infection. Mounting an immune response is known to have an energetic cost, hypothesized to result in trade offs between immunity and response to other stresses $[51,52,53]$. Increase in chill coma recovery time during infection may be contributed by insufficient energy stores.

Decline in negative geotactic ability, was also measured by how high a fly climbs in 10s post startle, in control and infected flies. In cold hardening lifespan and immunity is mainly affected [54] whereas an advantage of negative geotaxis assay is that the same set of flies were possibly used throughout the course of an infection. Hereby, both chill coma recovery and negative geotaxis could be useful in understanding the time course and degree to which different infections alter health in different genetic backgrounds of D. melanogaster. In addition to provide new measures for assessing health, these assays also suggest different pathological consequences of and metabolic up and downs that may occur over the course of an infection. Overall in brief, we can say that measures of health using these non-lethal behavioral assays may provide further insight into the mechanisms by which this model organism is able to maintain good health in facing of infections.

\section{Conclusion}

We demonstrated that how both chill coma recovery and negative geotaxis could be useful in deep understanding the time duration and different infections duration degree that results in structural changes of health in different genetic backgrounds of D. melanogaster. Health measurements using these pleiotropic assays may further provides different measures that how an organism behaves and shows its leverage after fungal infection.

\section{References}

[1] Sheldon, B. \& Verhulst, S. (1996) Ecological immunology:costly parasite defences and trade-offs in evolutionary ecology. Trends in Ecology and Evolution 11, 317-321.

[2] Schmid-Hempel, P. \& Ebert, D. (2003) On the evolutionary ecology of specific immune defence. Trends in Ecology and Evolution 18, 27-32.

[3] Medzhitov R, Schneider DS, Soares MP,(2012) Disease Tolerance as a Defense Strategy. Science335:936-941.

[4] Protzer U, Maini MK, Knolle PA (2012) Living in the liver: hepatic infections. 1-13.

[5] French SS, Denise Dearing M, Demas GE (2011) Leptin as a Physiological Mediator of Energetic Trade-Offs in Ecoimmunology: Implications for Disease. Integrative and Comparative Biology51:505-513.

[6] Mansueto P, Vitale G, Cascio A, Seidita A, Pepe I, et al. (2012) New Insight into Immunity and Immunopathology of Rickettsial Diseases. Clinical and Developmental Immunology 2012: 1-26.

[7] Riveron J, Boto T, Alcorta E (2009) The effect of environmental temperature on olfactory perception in Drosophila melanogaster. $J$ Insect Physiol 55:943-951, doi:10.1016/j.jinsphys.2009.06.009, pmid:19559705.CrossRefPubMedGoogle Scholar

[8] Newman MG, Castonguay LG, Borkovec TD, Molnar C. Integrative psychotherapy. In: Heimberg RG, Turk CL, Mennin DS, editors. Generalized anxiety disorder: Advances in research and practice. New York, NY: Guilford Press; 2004. pp. $320-350$.

[9] de Carvalho JF, Pereira RMR, Gershwin ME (2009) Hematopoietic cell transplants in autoimmunity. Isr Med Assoc J 11: 629-632.

[10] Schneider DS, Ayres JS (2008) Two ways to survive infection: what resistance and tolerance can teach us about treating infectious diseases. Nat Rev Immunol 8:889-895.

[11] Doucet et al., 2009 J.-L. Doucet, Y.L. Kouadio, D. Monticelli, P. Lejeune Enrichment of logging gaps with moabi (Baillonella toxisperma Pierre) in a Central African rain forest For. Ecol. Manage., 258 (2009), pp. 2407-2415

[12] Chambers MC, Schneider DS (2012) Pioneering immunology: insect style. Curr Opin Immunol 24: 10-14.

[13] Hamilos G, Samonis G, Kontoyiannis DP (2012) Recent Advances in the Use of Drosophila melanogaster as a Model to Study Immunopathogenesis of Medically Important Filamentous Fungi. International Journal of Microbiology 2012: 1-10.

[14] Boyer L, Paquette N, Silverman N, Stuart LM (2011) Advances in Experimental Medicine and Biology. 710: 29-36.

[15] Mckean KA, Yourth CP, Lazzaro BP, Clark AG (2008) The evolutionary costs of immunological maintenance and deployment. BMC Evol Biol 8: 76

[16] Linder JE, Promislow DEL (2009) Cross-generational fitness effects of infection in Drosophila melanogaster. Fly (Austin) 3: 143150 .

[17] Colinet H, Lee SF, Hoffmann A (2010) Functional characterization of the Frost gene in Drosophila melanogaster: importance for recovery from chill coma. PLoS ONE 5: e10925.

[18] Clark MS, Worland MR. How insects survive the cold: molecular mechanisms: a review. Comp Biochem Physiol B. 2008;178:917933. [PubMed]

[19] Landis GN, Abdueva D, Skvortsov D, Yang J, Rabin BE, et al. (2004) Similar gene expression patterns characterize aging and oxidative stress in Drosophila melanogaster. Proc Natl Acad Sci USA 101: 7663-7668.

[20] Driver CJ, Lamb MJ (1980) Metabolic changes in ageing Drosophila melanogaster. Exp Gerontol 15: 167-175.

[21] Le Bourg E, Lints FA (1984) A longitudinal study of the effects of age on spontaneous locomotor activity in Drosophila melanogaster. Gerontology 30: 79-86.

[22] Burger JMS, Promislow DEL (2006) Are functional and demographic senescence genetically independent? Exp Gerontol 41: 11081116.

[23] Macdonald SS, Rako L, Batterham P, Hoffmann AA (2004) Dissecting chill coma recovery as a measure of cold resistance: evidence for a biphasic response in Drosophila melanogaster. Journal of Insect Physiology 50: 695-700. 
[24] Goto SG, Udaka H, Ueda C, Katagiri C (2010) Fatty acids of membrane phospholipids in Drosophila melanogaster lines showing rapid and slow recoveryfrom chill coma. Biochemical and Biophysical Research Communications 391: $1251-1254$

[25] Chen C-P, Walker VK (1993) Cold-shock and Chilling Tolerance in Drosophila. Journal of Insect Physiology 40: 661-669.

[26] Llorens JV, Navarro JA, Martı 'nez-Sebastia 'n MJ, Baylies MK, Schneuwly S, etl al. (2007) Causative role of oxidative stress in a Drosophila model of Friedreich ataxia. FASEB J 21: 333-344.

[27] Hosamani R, Ramesh SR, Muralidhara (2010) Attenuation of rotenone-induced mitochondrial oxidative damage and neurotoxicty in Drosophila melanogaster supplemented with creatine. Neurochem Res 35: 1402-1412.

[28] Arking R, Wells RA. Genetic alteration of normal aging processes is responsible for extended longevity in Drosophila. Dev Genet 1990;11(2):141-148. [PubMed: 2116251]

[29] Le Bourg E, Massou I, Gobert V (2009) Cold stress increases resistance to fungal infection throughout life in Drosophila melanogaster. Biogerontology 10: 613-625.

[30] Le Bourg E (2007) Hormetic effects of repeated exposures to cold at young age on longevity, aging and resistance to heat or cold shocks in Drosophila melanogaster. Biogerontology 8: 431-444.

[31] Le Bourg E (2010) Combined effects of suppressing live yeast and of a cold pretreatment on longevity, aging and resistance to several stresses in Drosophila melanogaster. Biogerontology 11: 245-254.

[32] Miquel J, Lundgren PR, Bensch KG, Atlan H. Effects of temperature on the life span, vitality and fine structure of Drosophila melanogaster. Mech Ageing Dev 1976;5(5):347-370. [PubMed: 823384]

[33] Chavous DA, Jackson FR, O'Connor CM. Extension of the Drosophila lifespan by overexpression of a protein repair methyltransferase. Proc Natl Acad Sci U S A 2001;98(26):14814-14818. [PubMed:11742076]

[34] Mockett RJ, Orr WC, Rahmandar JJ, Sohal BH, Sohal RS. Antioxidant status and stress resistance in long- and short-lived lines of Drosophila melanogaster. Exp Gerontol 2001;36(3):441-463. [PubMed: 11250117]

[35] Kang HL, Benzer S, Min KT.(2002) Life extension in Drosophila by feeding a drug. Proc Natl Acad Sci U S A;99(2):838-843. [PubMed: 11792861]

[36] Ruan H, Tang XD, Chen ML, Joiner ML, Sun G, Brot N, Weissbach H, Heinemann SH, Iverson L, Wu CF, Hoshi T, Joiner MA. High-quality life extension by the enzyme peptide methionine sulfoxide reductase. Proc Natl Acad Sci U S A 2002;99(5):27482753. [PubMed: 11867705]

[37] Goddeeris MM, Cook-Wiens E, Horton WJ, Wolf H, Stoltzfus JR, Borrusch M, Grotewiel MS. Delayed behavioural aging and altered mortality in Drosophila beta integrin mutants. Aging Cell 2003;2(5):257-264. [PubMed: 14570233]

[38] Morrow G, Samson M, Michaud S, Tanguay RM. Overexpression of the small mitochondrial Hsp22 extends Drosophila life span and increases resistance to oxidative stress. Faseb J 2004;18(3):598-599. [PubMed: 14734639]

[39] Gargano JW, Martin I, Bhandari P, Grotewiel MS (2005) Rapid iterative negative geotaxis (RING): a new method for assessing age-related locomotor decline in Drosophila. Exp Gerontol 40: 386-395.

[40] Simon AF, Liang DT, Krantz DE. Differential decline in behavioral performance of Drosophila melanogaster with age. Mech Ageing Dev 2006;127(7):647-651. [PubMed: 16616306]

[41] Ayres JS, Schneider DS (2009) The role of anorexia in resistance and tolerance to infections in Drosophila. PLoS Biol 7: e1000150.

[42] Shirasu-Hiza MM, Dionne MS, Pham LN, Ayres JS, Schneider DS (2007)

[43] Interactions between circadian rhythm and immunity in Drosophila melanogaster. Curr Biol 17: R353-355.

[44] Medzhitov R, Schneider DS, Soares MP (2012) Disease Tolerance as a Defense Strategy. Science 335: 936-941.

[45] Macmillan HA, Sinclair BJ (2011) Mechanisms underlying insect chill-coma. Journal of Insect Physiology 57: 12-20.

[46] Zhang J, Marshall KE, Westwood JT, Clark MS, Sinclair BJ (2011) Divergent transcriptomic responses to repeated and single cold exposures in Drosophila melanogaster. Journal of Experimental Biology 214: 4021-4029.

[47] Xu J, James RR (2012) Temperature stress affects the expression of immune response genes in the alfalfa leafcutting bee, Megachile rotundata. Insect Molecular Biology 21: 269-280.

[48] Colinet H, Lee SF, Hoffmann A (2010) Knocking down expression of Hsp22 and Hsp23 by RNA interference affects recovery from chill coma in Drosophila melanogaster. Journal of Experimental Biology 213: 4146-4150.

[49] Udaka H, Ueda C, Goto SG (2010) Survival rate and expression of Heat-shock protein 70 and Frost genes after temperature stress in Drosophila melanogaster lines that are selected for recovery time from temperature coma. Journal of Insect Physiology 56: 1889-1894.

[50] Schwan WR, Goebel W (1994) Host cell responses to Listeria monocytogenes infection include differential transcription of host stress genes involved in signal transduction. Proc Natl Acad Sci USA 91: 6428-6432.

[51] De Gregorio E, Spellman PT, Tzou P, Rubin GM, Lemaitre B (2002) The Toll and Imd pathways are the major regulators of the immune response in Drosophila. EMBO J 21: 2568-2579.

[52] French SS, Denise Dearing M, Demas GE (2011) Leptin as a Physiological Mediator of Energetic Trade-Offs in Ecoimmunology: Implications for Disease.Integrative and Comparative Biology 51: 505-513.

[53] Ots I, Kerimov AB, Ivankina EV, Ilyina TA, Horak P (2001) Immune challenge affects basal metabolic activity in wintering great tits. Proceedings of the Royal Society B: Biological Sciences 268: 1175-1181.

[54] Nelson RJ, Demas GE (1996) Seasonal changes in immune function. Q Rev Biol 71: 511-548.

[55] Le Bourg E (2010) A cold stress applied at various ages can increase resistance to heat and fungal infection in aged Drosophila melanogaster flies. Biogerontology. 